\title{
Composition en acides gras des œufs : intérêt nutritionnel et valeur santé
}

\author{
Gérard PIERONI \\ Thierry C. COSTE
}

Applications santé des lipides (ASL),

Bioparc Vichy,

03270 Hauterive,

France

<gerard.pieroni@asl-lipides.com>

Les œufs de consommation sont quasi exclusivement des œufs de poule. Ils présentent une excellente valeur alimentaire tant du point de vue de leur composition équilibrée en acides aminés que du point de vue de leur composition en lipides, en minéraux et en vitamines (Bourre, 2005 ; Cotterill et al., 1977 ; Nys et Sauveur, 2004). En France, leur consommation moyenne est de l'ordre de cinq œufs par semaine et par personne (ITAVI, 2010). De façon faible mais significative, ils contribuent ainsi à l'alimentation de la population. Bien que de composition globale remarquablement stable, certains des composés de l'œuf présentent une plasticité de leur composition. Ainsi, la modification de la teneur relative des œufs en acides gras, et tout particulièrement en acides gras essentiels et leurs dérivés, a fait I'objet de nombreux travaux depuis plus d'un siècle (Henriques et Hansen, 1903). La présente revue se propose de rappeler la composition lipidique des œufs et de faire le point sur les travaux concernant leur enrichissement en certains acides gras et les perspectives qui en découlent en termes d'impact potentiel sur la santé.

\section{Guf « moyen ", aspects quantitatifs}

On peut considérer que le poids moyen d'un œuf de poule est de $60 \mathrm{~g}$ avec une variabilité (deux écarts-types) de $10 \%$ environ, les extrêmes étant 35 à $70 \mathrm{~g}$. Le jaune représente environ $29 \%$ de la masse de l'œuf $(17,4 \mathrm{~g})$, les lipides du jaune : $34 \pm 2 \%$ de la masse du jaune, soit $5,9 \mathrm{~g}$ environ et les acides gras $83 \%$ de la masse des lipides, soit 4,9 g. Les lipides se partagent entre triacylglycérols pour $67 \%$, soit $3,9 \mathrm{~g}$; phospholipides : $28 \%$, soit $1,65 \mathrm{~g}$ et

\begin{abstract}
The average composition in lipids and fatty acids of the hen's egg is reported. The fact that the egg is a source of phospholipids is pointed out. The enrichment in essential fatty acids in response to different dietary lipids is reported with a focus on enrichment with omega 3 fatty acids. The results of the different approaches of enrichment through seeds oils, marine oils or both are described. The potential interest of using omega 3 enriched egg, specially DHA enriched egg, in human nutrition is documented through the specific properties of DHA-bearing egg phospholipids in terms of stability to oxidation, better bioavailability and specific physiological effects.
\end{abstract}

Key words: egg, fatty acids, phospholipids, composition, n-3, DHA cholestérol : 300 mg, environ. Les acides gras portés par les triacylglycérols représentent $3,7 \mathrm{~g}$, alors que ceux portés par les phospholipides représentent $1,2 \mathrm{~g}$.

\section{Composition en acides gras}

La variabilité de la composition en acides gras des lipides de l'œuf en réponse aux variations de l'alimentation et aux conditions d'élevage fait qu'il est impossible de donner une composition précise moyenne en acides gras. Néanmoins, il existe quelques points forts qui apparaissent dans le tableau 1.

On peut remarquer que la plus grande variabilité de composition est associée à l'acide linoléique ainsi qu'à l'acide alpha-linolénique et à ses dérivés à 20 atomes de carbone et plus.
Ce sont ces mêmes acides gras qui répondent aux modifications des apports alimentaires (liang et al., 1991). En termes quantitatifs, les acides gras saturés représentent environ un tiers des acides gras, et les acides gras monoinsaturés environ un acide gras sur deux. L'apport en acide linoléique d'un œuf moyen est de $0,65 \mathrm{~g}$ et, en acides gras oméga- 3 , de $0,07 \mathrm{~g}$ environ.

\section{Répartition des acides gras par espèce lipidique}

Les acides gras de l'œuf ne se répartissent pas de façon identique entre les triacylglycérols et les phospholipides, et cela aussi bien en termes de nature des acides gras déposés qu'en termes de régiosélectivité sur le glycérol (tableau 2).
Tableau 1. Composition en acides gras, (\% p/p), des lipides totaux de l'œuf.

\begin{tabular}{|lll|}
\hline Acides gras & Moyenne relative (\%) & Fourchette (\%) \\
\hline Acides gras saturés (AGS) & 35 & {$[32-39]$} \\
\hline Acides gras mono-insaturés (ACMI) & 46 & {$[43-51]$} \\
\hline Acide linoléique $(\mathrm{C} 18: 2 \mathrm{n}-6, \mathrm{LA})$ & 14 & {$[7-18]$} \\
\hline Acide alpha-linolénique $(\mathrm{C} 18: 3 \mathrm{n}-3, \mathrm{ALA})$ & 0,8 & {$[0,1-1,5]$} \\
\hline Acide arachidonique $(\mathrm{C} 20: 4 \mathrm{n}-6, \mathrm{AA})$ & 2 & {$[1-3]$} \\
\hline Acide docosahexaénoïque $(\mathrm{C} 22: 6 \mathrm{n}-3, \mathrm{DHA})$ & 0,9 & {$[0,5-1,5]$} \\
\hline
\end{tabular}

Tableau 2. Composition en acides gras, (\% p/p).

\begin{tabular}{|llllllll|}
\hline Acides gras & AGS & AGMI & C18:2 & C18:3 & C20:4 & C20:5 & C22:6 \\
\hline Triglycérides & 34 & 55 & 10 & 0,6 & tr & tr & tr \\
\hline Phospholipides & 50 & 31 & 9 & 0,5 & 5 & 0,4 & 2,5 \\
\hline
\end{tabular}

tr : traces. 
Les triacylglycérols de l'œuf sont caractérisés par une localisation préférentielle de l'acide palmitique en position sn-1 du glycérol, tandis que l'acide oléique se partage de façon équivalente entre les positions sn-2 et sn-3 (Kuksis, 1992). Les acides linoléique et alphalinolénique occupent préférentiellement la position sn-2 (Schreiner et al., 2004).

Les acides gras polyinsaturés (AGPI) à 20 atomes de carbone et plus (AGPI-LC) sont localisés quasi exclusivement dans les phospholipides. Les phospholipides comportent une proportion plus élevée d'acides gras saturés (un acide gras sur deux environ) que les triacylglycérols (un acide gras sur trois environ).

Dans les phosphatidylcholines, les acides gras saturés sont préférentiellement localisés en position sn-1 (Kuksis, 1992), pour plus de $90 \%$ (Schreiner et al., 2004). II en est de même pour les phosphatidyléthanolamines, ces dernières présentant une proportion plus importante en position sn-1 d'acide stéarique par rapport à l'acide palmitique.

\section{Modification de la composition en acides gras de l'œuf}

Le métabolisme des acides gras chez la poule est très actif, et ce d'autant plus que la teneur en lipides de la ration journalière est faible. II conduit principalement à la synthèse endogène d'acides palmitique et oléique (Balnave, 1975). Les AGPI tels l'acide linoléique et l'acide alpha-linolénique doivent être apportés par I'alimentation, ils sont, au même titre que pour l'Homme, des acides gras essentiels. Ce sont ainsi les teneurs de ces AGPI et celles de leurs dérivés métaboliques qui sont susceptibles de répondre aux modifications de l'alimentation (Jiang et al., 1991). De façon générale, la composition en acides gras de l'œuf est conditionnée à la fois par la nature et l'état du cheptel, les conditions d'élevage et par l'alimentation des pondeuses.

\section{Facteurs de modification non alimentaires}

\section{Souche et âge des pondeuses}

II existe relativement peu d'études qui documentent les effets de la souche de pondeuse sur l'enrichissement en acides gras. Néanmoins, dans leur ensemble, les différentes études concourent à accorder un faible rôle à la race de la poule pondeuse sur l'impact de la nutrition sur la composition en acides gras (Ahn et al., 1995).

\section{Durée d'application du régime}

On peut considérer que les pondeuses répondent sous deux semaines à une modification de la composition en acides gras de leur aliment, l'effet maximal étant atteint vers trois-quatre semaines.

\section{Facteurs de modifications alimentaires}

Compte tenu que seule la composition en AGPI de l'œuf peut présenter des variations relatives importantes, la quasi-totalité des études s'est focalisée sur ce point. D’une façon générale, I'enrichissement en AGPI se fait principalement en substitution de l'acide oléique et secondairement des acides à 16 atomes de carbone (Thapon et Bourgeois, 1994).

\section{Apports en AGPI oméga 6}

Les teneurs du jaune en acide linoléique peuvent être multipliées par 2 par simple apport $d^{\prime}$ huile de soja dans le régime. Le pourcentage maximum en acide linoléique qui peut être atteint dans les acides gras est voisin de 30 $35 \%$ (Tapon et Bourgeois, 1994), soit 1,6 g environ par œuf. Cet enrichissement ne $s^{\prime}$ accompagne pas d'un accroissement proportionnel du pourcentage en acide arachidonique qui demeure relativement constant. Ainsi, l'enrichissement de l'œuf en acides gras oméga-6 présente un intérêt limité dans la mesure où, d'une part, les apports en acide linoléique sont considérés comme excessifs dans notre alimentation et où, d'autre part, le métabolisme de l'animal n'apporte aucun bénéfice qualitatif en termes de nature des acides gras de cette famille.

\section{Apports en AGPI oméga-3}

L'enrichissement de l'aliment pondeuse en acides gras oméga-3 peut être réalisé à partir de sources uniquement végétales (Lin et al., 1991), c'est-à-dire à partir d'acide alphalinolénique, les plantes ne synthétisant pas ou très peu les AGPI-LC; ou bien d'algues sélectionnées qui peuvent constituer des sources riches en acides gras oméga-3 (Herber et Van Elswyk, 1996), en C20 et C22, bien que les plus utilisées soient sélectionnées pour être une source unique d'acide docosahexaénoïque (DHA ; C22:6). Par ailleurs, les huiles d'origine marine (poissons, mammifères marins, crustacés), qui apportent acide eïcosapentaénoïque (EPA; C20:5) et DHA principalement, constituent une autre source (tableau 3 ; Huang et al., 1990 ; Lin et al., 1991).

Chez I'animal I'acide alpha-linolénique subit des réactions d'élongation et de désaturation conduisant principalement à I'EPA, à I'acide docosapentaénoïque (DPA; C22:5 n-3) et à des quantités limitées de DHA. Cette situation est tout à fait comparable à celle décrite chez I'Homme (Burdge et Calder, 2005 ; Brenna et al., 2009). Dans le cas d'apport d'EPA et de DHA, I'EPA est partiellement bêta-oxydé et un enrichissement préférentiel en DHA est observé.

En alimentation animale, les principales sources d'acide alpha-linolénique sont le colza, le soja et le lin. Des résultats variables ont été observés suivant la forme d'apport : huile, graine, traitement des graines et bien sûr, niveau d'incorporation, valeur énergétique de l'aliment, durée d'application du régime. Les huiles de poisson et les extraits d'algues sont les sources les plus communes d'AGPI-LC oméga-3 : EPA, DPA et DHA.

Tableau 3. Composition en acides gras, $(\%$ p/p), des lipides totaux de l'œuf en réponse à différents apports en acides gras $n-3$.

\begin{tabular}{|c|c|c|c|c|c|c|c|}
\hline \multirow[t]{2}{*}{ Acides gras } & \multirow{2}{*}{$\begin{array}{l}\text { AGS } \\
\Sigma\end{array}$} & \multirow{2}{*}{$\begin{array}{l}\text { AGMI } \\
\Sigma\end{array}$} & \multicolumn{2}{|c|}{ AGPI n-6 } & \multicolumn{3}{|c|}{ AGPI n-3 } \\
\hline & & & C18:2 & C20:4 & C18:3 & C20:5 & C22:6 \\
\hline Alimentation standard & 35 & 46 & 14 & 2 & 0,8 & - & 0,9 \\
\hline \multicolumn{8}{|l|}{ Huile végétale (\% p/p) } \\
\hline Soja $(10 \%)^{a}$ & 36 & 37 & 22 & 2,1 & 1,3 & - & 1,2 \\
\hline $\operatorname{Lin}(10 \%)^{a}$ & 37 & 39 & 9,3 & 0,8 & 9,1 & 0,4 & 1,9 \\
\hline \multicolumn{8}{|l|}{ Huile marine $(\% p / p)$} \\
\hline Menhaden ${ }^{c}(2 \%)^{b}$ & 38 & 41 & 14 & 1,3 & 0,5 & 0,4 & 4,2 \\
\hline Menhaden $^{c}(10 \%)^{a}$ & 43 & 44 & 1,9 & 0,7 & 0,3 & 2,5 & 6,7 \\
\hline
\end{tabular}

Alimentation standard : valeurs moyennes tirées du Tableau 1 « œuf moyen ».

a Lin et al., 1991.

${ }^{b}$ Huang et al., 1990.

cMenhaden : Brevoortia tyrannus, forme d'alose. 
L'apport d'acide alpha-linolénique dans I'alimentation des pondeuses conduit à un enrichissement correspondant relativement proportionnel dans les acides gras des lipides totaux de l'œuf. Les valeurs présentées sur le tableau 3 montrent que l'incorporation d'une source d'acide alpha-linolénique dans l'alimentation des pondeuses permet d'accroître de façon relativement proportionnelle la teneur en acide alpha-linolénique dans les lipides de I'œuf, jusqu'à 300-400 mg par œuf. En présence de $15 \%$ de graines de lin dans l'aliment, on peut observer l'incorporation jusqu'à près de 450 mg d'acide alpha-linolénique par œuf (Jia et al., 2008).

En ce qui concerne le DHA, les quantités accumulées plafonnent très rapidement en fonction des quantités d'acide alpha-linolénique apportées par l'alimentation, en général le taux maximal obtenu ne dépasse pas $2 \%$ des acides gras des lipides totaux, soit environ $100 \mathrm{mg}$ par œuf (Jia et al., 2008). Les rapports DHA/EPA sont de I'ordre de 4 à 5 dans le cas du lin.

L'apport d'huile de poisson ou bien de poudre $d$ 'algues spécifiquement enrichies en DHA se traduit par un accroissement proportionnel du DHA dans les lipides totaux de I'œuf. Les quantités accumulées peuvent dépasser les $300 \mathrm{mg}$ de DHA par œuf. Les rapports DHA/EPA peuvent atteindre des valeurs de 5 à 9 (Grune et al., 2001), ce qui, compte tenu que I'on n'observe pas d'enrichissement en acide alpha-linolénique et que les taux de DPA sont comparables à ceux de I'EPA, conduit à un enrichissement relativement spécifique en DHA parmi la famille des acides gras oméga 3.

L'incorporation de l'acide alpha-linolénique se fait très majoritairement au niveau des triglycérides, alors que l'incorporation du DHA, du DPA et de l'EPA est essentiellement observée au niveau des phospholipides, couramment à plus de $80 \%$ et tout particulièrement en position sn-2 des phospholipides, à plus de $90 \%$, DPA et EPA ont un comportement comparable, légèrement moins marqué (Schreiner et al., 2004). L'enrichissement en DHA, DPA et EPA des différentes fractions phospholipidiques ne se fait pas de façon égale. Les phosphatidyléthanolamines présentent des teneurs 3 à 4 fois plus élevées en ces acides gras que les phosphatidylcholines.

\section{Stabilité à l'oxydation}

En accord avec le fait que les lécithines sont reconnues comme des agents antioxydants, les AGPI associés aux phospholipides d'œuf présentent, comparativement aux mêmes acides gras sous forme de triacylglycérols ou éthyl esters, une stabilité vis-à-vis de l'oxydation, très significativement supérieure (figure 1 ; Song et al., 1997).

\section{Biodisponibilité accrue du DHA sous forme de phospholipide}

La biodisponibilité d'un acide gras peut s'évaluer par sa capacité à se retrouver dans les lipides plasmatiques, les différents acides gras alimentaires étant généralement très bien absorbés, les différences observées ne peuvent être que marginales. En revanche, lorsque la biodisponibilité du DHA est appréciée en termes d'accrétion dans différents organes, on peut observer que la forme phospholipide est plus efficace que les formes triacylglycérol et éthyl ester (tableau 4, Valenzuela et al., 2005).

\section{Effets physiologiques particuliers liés à la forme d'apport du DHA}

Dans un modèle d'hypoxie expérimentale chez le rat, la supplémentation avec du DHA sous forme de triacylglycérols d'algues ou bien de phospholipides d'œuf conduit à des réponses physiologiques différentes. Alors que la forme $d^{\prime}$ apport triacylglycérol ne se différencie pas du contrôle, I'apport de DHA sous forme de phospholipides d'œuf permet une conservation partielle des capacités respiratoires visualisées à la fois en termes d'endurance à l'effort et en termes d'activité des paramètres de la chaîne respiratoire (figure 2, Tardieu et al., 2009).

\section{Conclusion}

En France, les œufs apportent un maximum de $5 \%$ des lipides alimentaires, ils constituent ainsi une source modeste, mais non négligeable des apports lipidiques moyens évalués à $89,3 \mathrm{~g} / \mathrm{j}$ chezl'adulte (INCA 2, 2009). Quatrevingt-trois pour cent de ces lipides sont des acides gras et un quart de ces derniers sont portés par des phospholipides. Ces mêmes phospholipides sont associés à plus de $80 \%$ aux AGPILC, acide arachidonique pour les acides gras

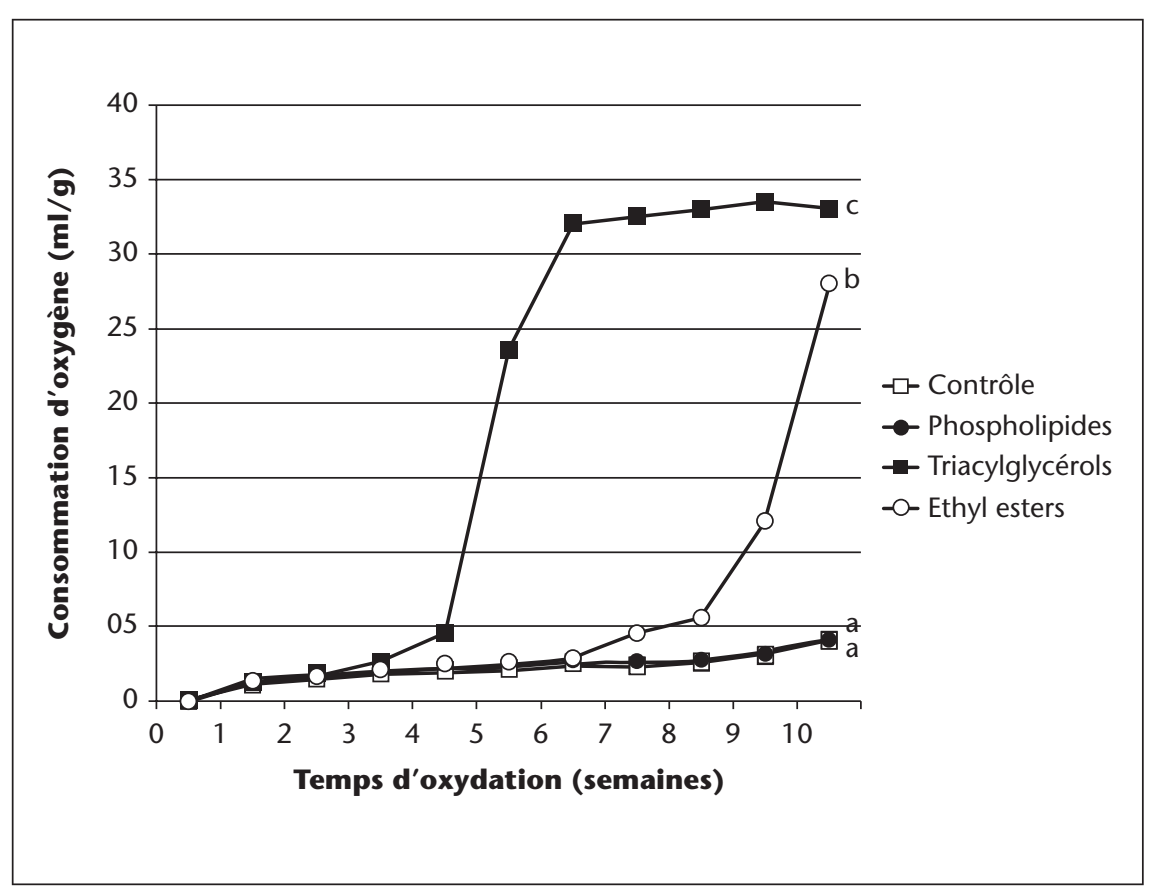

Figure 1. Stabilité à l'oxydation de divers lipides contenant $10 \%$ molaire de DHA (adapté de Song et al., 1997).

Tableau 4. Formes d'apport en DHA et accrétion tissulaire (D'après Valenzuela et al., 2005).

\begin{tabular}{|lllll|}
\hline $\begin{array}{l}\text { DHA } 8 \mathbf{~ m g} / \mathbf{k g} \\
\mathbf{4 0} \text { jours rattes }\end{array}$ & $\begin{array}{l}\text { Plasma } \\
(\boldsymbol{\mu g} / \mathbf{m L})\end{array}$ & $\begin{array}{l}\text { Erythrocyte } \\
\mathbf{m g} / \mathbf{g} \text { PL }\end{array}$ & $\begin{array}{l}\text { Tissu hépatique } \\
\mathbf{m g} / \mathbf{g} \text { PL }\end{array}$ & $\begin{array}{l}\text { Tissu adipeux } \\
\mathbf{m g} / \mathbf{g} \text { lipides }\end{array}$ \\
\hline Huile d'olive & 80 & 12,5 & 45 & 4,5 \\
\hline Phospholipides & $150(+90 \%)$ & $27,5(+120 \%)$ & $90(+100 \%)$ & $9(+100 \%)$ \\
\hline Triacylglycérols & $145(+80 \%)$ & $22,5(+80 \%)$ & $70(+55 \%)$ & $7(+55 \%)$ \\
\hline Ethyl esters & $150(+90 \%)$ & $17,5(+40 \%)$ & $40(-10 \%)$ & $5(+10 \%)$ \\
\hline
\end{tabular}




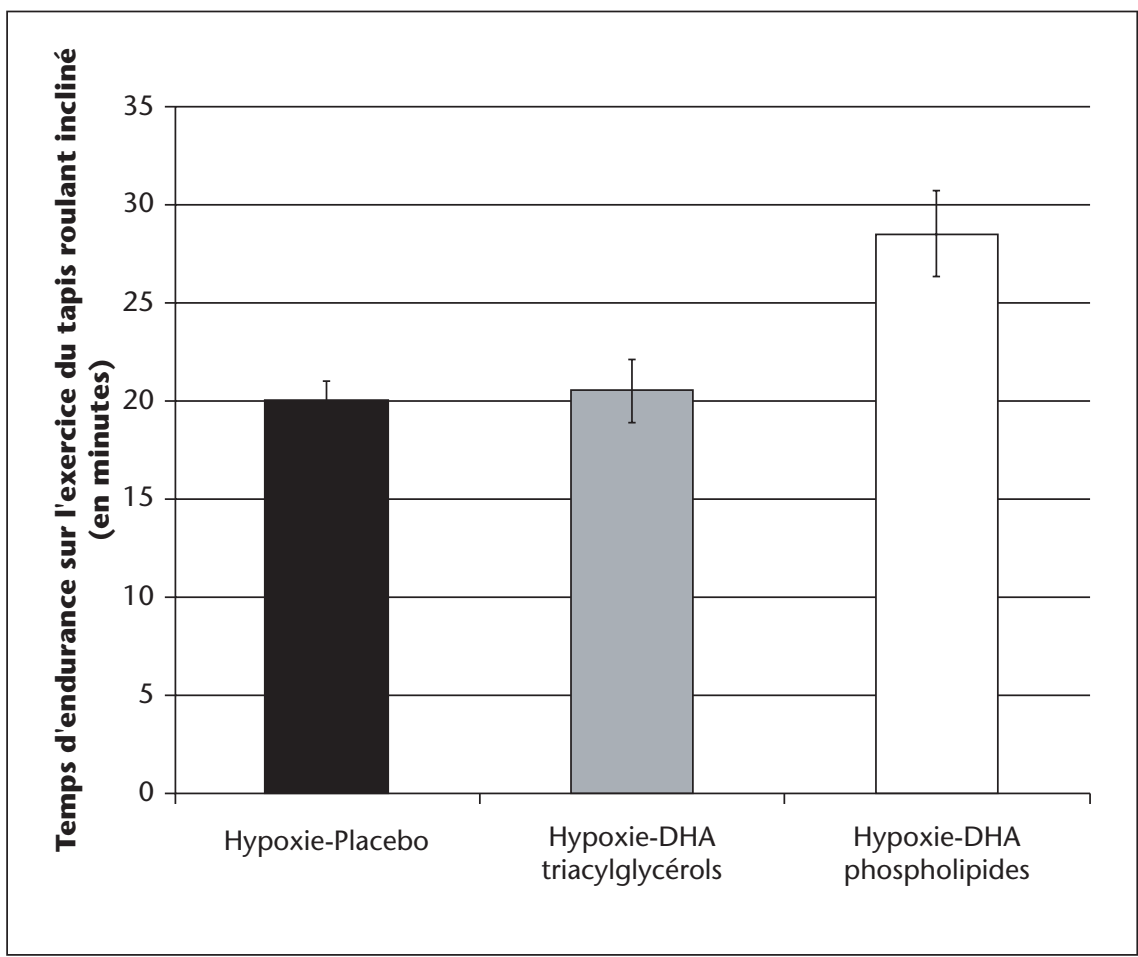

Figure 2. Endurances à l'effort comparées de rats après deux semaines d'hypoxie suite à 12 semaines de trois régimes différents : contrôle, DHA-triacylglycérols, DHA-phospholipides (Tardieu et al., 2009).

oméga-6, et acide docosahexaénoïque (DHA), pour les acides gras oméga 3, en particulier. Ainsi, les lipides de l'œuf sont très majoritairement le véhicule d'AGPI-LC sous forme de phospholipides. Cette originalité démarque I'œuf comme source d'AGPI-LC par rapport aux huiles d'origine marine dont les triacylglycérols portent les mêmes acides gras. Ces derniers présentent des propriétés remarquables de stabilité vis-à-vis de l'oxydation lorsqu'ils sont les constituants de phospholipides, cela en comparaison des formes triacylglycérol et éthyl ester. À ces propriétés de stabilité s'ajoute une accrétion accrue dans les tissus animaux de ces mêmes acides gras lorsqu'ils sont véhiculés par des phospholipides. II peut en résulter des effets physiologiques bénéfiques ou non suivant que l'acide gras apporté, le DHA en l'occurrence, l'est sous forme de phospholipide ou de triacylglycérol. L'ensemble de ces constatations doit conduire à considérer les lipides de l'œuf comme une source particulière d'ACPI-LC dont les effets sur la santé demeurent encore à être évalués. Dans cette perspective, le développement d'œufs spécifiquement enrichis en acides gras oméga-3, et en DHA en particulier, dont une partie de la population ne consomme qu'une fraction des apports nutritionnels conseillés, devrait constituer un moyen de recherche intéressant devant déboucher sur l'objectivation d'effets santé clairement identifiés et quantifiés. La validation de

ces effets sur la santé devrait conduire au développement d'œufs spécifiquement enrichis en certains acides gras et en DHA en particulier.

\section{RÉFÉRENCES}

Ahn DU, Sunwoo HH, Wolfe FH, Sim JS. Effect of dietary alpha-linolenic acid and strain of hen on the fatty acid composition, storage stability, and flavor characteristics of chicken eggs. Poult Sci $1995 ; 74$ : 1540-7.

Balnave $D$. The influence of essential fatty acids and food restriction on the specific activities of hepatic lipogenic and glutamate-metabolizing enzymes in the laying hen. Br J Nutr $1975 ; 33: 439-45$.

Bourre JM. Dossier scientifique sur l'œuf. Comité national pour la promotion de l'œuf. http://www. oeufs-asso.com/imgs/communique/

061221171608_du-neuf-sur-l-oeuf-.doc

(dernier accès le 23/01/2010) 2005.

Brenna JT, Salem Jr N, Sinclair AJ, Cunnane SC. ISSFAL. Alpha-linolenic acid supplementation and conversion to $n-3$ long-chain polyunsaturated fatty acids in humans. Prostaglandins Leukot Essent Fatty Acids $2009 ; 80: 85-91$.

Burdge GC, Calder PC. Conversion of alpha-linolenic acid to longer-chain polyunsaturated fatty acids in human adults. Reprod Nutr Dev 2005 ; 45 : 581-97.

Cotterill OJ, Marion WW, Naber EC. A nutrient reevaluation of shell eggs. Poult Sci 1977; 56: 1927-34.
Grune T, Krämer K, Hoppe PP, Siems W. Enrichment of eggs with $n-3$ polyunsaturated fatty acids: effects of vitamin E supplementation. Lipids $2001 ; 36$ : 833-8.

Henriques V, Hansen C. Skand Arch Physiol 1903 ; $14: 390-7$.

Herber SM, Van Elswyk ME. Dietary marine algae promotes efficient deposition of n-3 fatty acids for the production of enriched shell eggs. Poult Sci 1996; $75: 1501-7$.

Huang Z, Leibovitz H, Lee CM, Millar R. Effect of dietary fish oil on omega-3 fatty acids levels in chicken eggs and thigh flesh. J Agric Food Chem 1990; 38 : 743-7.

INCA 2: synthèse de l'étude individuelle nationale des consommations alimentaires 2 (2006-2007). http:// www.afssa.fr/Documents/PASER-Sy-INCA2EN.pdf (dernier accès le 23/01/2010).

ITAVI. Institut technique de I'aviculture 2010. www. itavi.asso.fr/ (dernier accès le 23/01/2010).

Jia W, Slominski BA, Guenter W, Humphreys A, Jones $O$. The effect of enzyme supplementation on egg production parameters and omega- 3 fatty acid deposition in laying hens fed flaxseed and canola seed. Poult Sci $2008 ; 87$ : 2005-14.

Jiang ZR, Ahn DU, Sim JS. Effects of feeding flax and two types of sunflower seeds on fatty acid compositions of yolk lipid classes. Poult Sci 1991; 70 : 2467-75.
Kuksis A. Yolk lipids. Biochim Biophys Acta 1992 ; 1124 : 205-22.

Lin DS, Connor WE, Anderson G). The incorporation of $n-3$ and $n-6$ essential fatty acids into the chick embryo from egg yolks having vastly different fatty acid compositions. Pediatr Res $1991 ; 29: 601-5$.

Nys $Y$, Sauveur B. Valeur nutritionnelle des œufs. INRA Prod Anim $2004 ; 17$ : 385-93.

Schreiner M, Hulan HW, Razzazi-Fazeli E, Böhm J, Iben C. Feeding laying hens seal blubber oil: effects on egg yolk incorporation, stereospecific distribution of omega- 3 fatty acids, and sensory aspects. Poult Sci $2004 ; 83$ : 462-73.

Song JH, Inoue $\mathrm{Y}$, Miyazawa T. Oxidative stability of docosahexaenoic acid-containing oils in the form of phospholipids, triacylglycerols, and ethyl esters. Biosci Biotechnol Biochem 1997 ; 61 : 2085-8.

Tardieu C, Chaté V, Guzun R, et al. Loss of body mass and exercise endurance under hypoxic conditions is reduced with glycerophospholipids enriched in docosahexaenoic acid (GPL-DHA) supplementation in rodents. Clin Nutr $2009 ;$; 4 : 6.

Thapon JL, Bourgeois CM. L'œuf et les ovoproduits. Paris: Tech et Doc, Lavoisier, 1994, p 78.

Valenzuela A, Nieto S, Sanhueza J, Nunez MJ, Ferrer C. Tissue accretion and milk content of docosahexaenoic acid in female rats after supplementation with different docosahexaenoic acid sources. Ann Nutr Metab $2005 ; 49$ : 325-32. 